\title{
THE DETERMINANTS OF CLOUD COMPUTING ADOPTION IN SAUDI ARABIA
}

\author{
Abdullah Alhammadi, Clare Stanier, Alan Eardley \\ Faculty of Computing, Engineering and Sciences, Staffordshire University, \\ Beaconside, Stafford, Staffordshire, United Kingdom ST18 0AD \\ a.alhammadiestaffs.ac.uk, c.stanier@staffs.ac.uk, \\ w.a.eardley@staffs.ac.uk
}

\begin{abstract}
There is a large volume of published studies investigating the factors that affect cloud adoption. However, there are very few studies which investigate cloud computing adoption in technologically developing countries and one focus of the research was to examine whether the factors which influence cloud computing adoption in technologically developed countries also apply in technologically developing countries. The research presented in this paper builds on the diffusion of innovation theory (DOI) and the Technology-organisation-environment (TOE) framework in order to investigate the factors which influence cloud adoption. Fourteen hypothesis were developed from the literature on cloud adoption and were examined in the research. Data was collected by using a web-based questionnaire and was analysed using a range of statistical measures. This paper discusses the design and implementation of the study, the data analysis and conclusions from the analysis and compares the findings of this study with the findings of similar studies in technologically developed countries. The study shows that there are some similarities as well as some differences in the factors that affect cloud adoption between technologically developed countries and technologically developing countries.
\end{abstract}

\section{KEYWORDS}

Cloud computing adoption, Diffusion of innovation (DOI), Technology-organisationenvironment (TOE)

\section{INTRODUCTION}

Cloud computing has been described as the next generation model of computing [1]. The cloud computing was defined by National Institute of Standards and Technology NIST as a model for enabling convenient, on-demand network access to a shared pool of configurable computing resources [...] that can be rapidly provisioned and released with minimal management effort or service provider interaction" (NIST, 2011). Cloud computing has four deployment models namely public cloud, private cloud, hybrid cloud and community cloud. Enterprise spending on cloud computing is increasing at five times the rate of traditional IT systems (cited by [2]. However, cloud computing adoption rates vary between technologically developed countries and technologically developing countries [3]. The literature on Cloud Computing adoption focuses primarily on adoption in technologically developed countries. The literature does not include any empirical study carried out to investigate cloud adoption in the Kingdom of Saudi Arabia (KSA)

David C. Wyld et al. (Eds) : CSEN, AISO, NCWC, SIPR - 2015

pp. 55-67, 2015. C CS \& IT-CSCP 2015

DOI : $10.5121 /$ csit.2015.51406 
at the organisational level. There is only one study which investigates cloud adoption in KSA [4] and this focused on the user acceptance of cloud computing at an individual level while factors related to an organisational level were ignored. This study discussed the factors that affect cloud computing decision by integrating DOI and TOE framework.

\section{THEORETICAL BACKGROUND}

The conceptual model that is developed in this paper is based on concepts derived from the diffusion of innovation (DOI) theory and Technology, Organisation, Environment (TOE) framework. DOI, first proposed by Rogers (1962), is concerned with the way in which new ideas are adopted within organisations over time and how ideas influence change within organisations. DOI identifies five factors that affect the adoption of new ideas: relative advantage, compatibility, complexity, trialability and observability [5]. TOE framework was developed by Tornatzky and Fleischer in 1990 to investigate innovation adoption at organisation level [6], [7] and the framework provides a holistic picture of the factors that influence the adoption of technology [7], [8]. TOE categorises the factor which influence technology adoption in organisation into three categories namely, technological context, organisation context, and environmental context [8]. It has been argued that the use of these three elements gives the TOE framework the advantage over other technology adoption theories in studying technology use, adoption and the value added from technology innovation [7]. Numerous studies have attempted to investigate the adoption of new technologies from both individual and organizational perspective [9]-[11]. These studies have tried to identify factors which influence technology adoption and the relationship between these factors and technology adoption [7]. Technology adoption theory plays an important role in investigation the adoption of technology in different disciplines such as E-commerce, RFID, ERP [12]-[16]. Several studies have used outsourcing theories to investigate cloud adoption [17] . Technology adoption theories have been used to support the investigation of cloud computing adoption in a number of studies $[10,18,19]$. In this paper, we use a DOI/TOE approach as the focus of the study is on the adoption of cloud computing at organisational level.

From the literature on cloud computing adoption, we identified 14 factors which influence organisational decisions about the adoption of cloud computing. From these factors, we developed hypothesis as to the way in which the factors affect the adoption of cloud computing. Following TOE, we group these hypothesis into the TOE categories of technology context, organisational context and environment context and we also use a fourth category, the DOI category, which address elements identified though analysis based on DOI. The second phase of the research evaluated the hypothesis through use of a questionnaire. This section describes the categories used and way in which hypothesis were developed from the factors identified in the literature.

\subsection{Technology context}

The Technology context refers to a "comprised of the variables that influence an individual, an organization, and an industry's adoption of innovation" [7] 


\subsubsection{Technology readiness}

Cloud computing is a relatively new model of IT service delivery model. Thus, the technology context is a very important determinant to investigate in relation to the adoption of cloud computing. Technology readiness is described as the IT infrastructure available to an enterprise to obtain cloud services and human resources that can manage cloud services [10]. We therefore identify as hypothesis 1 :

H1: technology readiness positively influences cloud computing adoption.

\subsubsection{Security}

Security is seen as one of the highest risk elements in the adoption of cloud computing [18]-[20] although it has also been argued that there is no relationship between security and cloud adoption [10]. This inconsistency is tested by hypothesis $2:$.

H2: security concerns negatively influence cloud computing adoption.

\subsubsection{Technical barrier}

Technical issues such as the complexity of existing IT systems, portability and interoperability and vendor lock-in have been identified as possible barriers to the adoption of cloud computing [21].

H3: technology barrier negatively influence cloud computing adoption.

\subsection{Organisational context}

The organisational context refers to the characteristics of the organisation and its internal resources [9], [22]. Organisation characteristics include organisation size, status, industry, and scope. While the internal resources include knowledge capability, top management support and organisation readiness [7]. Cloud computing has technological as well as organisational implication, this makes the organisational context a key determinant of cloud adoption.

\subsubsection{Enterprise size}

Enterprise size is considered to be one of the main factors influencing technical innovation [9], [23]-[25]. It has been argued that large enterprises are more likely to adopt newer technologies such as ERP and e-commerce [24], [26]. The main reasons given being that larger enterprise have greater organisational and financial resources. However, the financial model of cloud computing services makes it attractive to SMEs meaning that enterprise size is one of the determinants to investigate in relation to cloud computing adoption.

H4: enterprise size has a positive influence to adopt cloud computing services. 


\subsubsection{Top management support}

Top management support refers to the decision makers who influence the adoption of innovation [27] and is which described by [28] as goal specificity, resource management, and commitment. Thus, the role of top management support is crucial to success the adopting of new technology. Therefore, Top management support is regarded in many studies as an important factor influencing the adoption of technology innovation [29]-[31].

H5: top management support has a positive impact on cloud adoption.

\subsubsection{Organisation readiness}

Organisation readiness aims to measure if the organisation has the capability to adopt the innovation [6] and is defined as the availability of human, technology and financial resources to adopt cloud computing [6], [13], [32]. Knowledge about cloud computing and the attitudes towards using the technology are an important factors in the adoption of cloud computing.

H6: organisation readiness has a positive impact on cloud adoption.

\subsubsection{Enterprise status}

Enterprise status is defined in this study as having either status as an establish company or as a Start-up company. There is no empirical study in the literature which investigates the impact of enterprise status impact on technology adoption in general and cloud adoption specifically. However, some studies have linked enterprise status and cloud computing adoption [33], [34].

H7: enterprise status has a positive impact on cloud adoption.

\subsubsection{Industry sector}

IT has a major impact on how enterprises manages their business but the role of IT in an organisation differs, depending on the sector [35]. Moreover, technology adoption varies between sectors, for example, the largest user of technology is the financial sector [25]. However, sensitivity of financial data means that the financial sector may be more cautious when adopting cloud computing [36], [37].

H8: industry sector has a positive impact on cloud adoption.

\subsection{Environmental context}

Environmental context refers to the external factors that influence the adoption of technology includes government regulation and initiative, service providers and competitors [7]. This study will investigate the following factors in terms of environmental context; competitive pressure, external support and government support. 


\subsubsection{Competitive pressure}

Competitive pressure may be a factor which influences the adoption of cloud computing although there is no consensus on this issue. It has been argued that competitive pressure is very influential in the adoption of technology in general [6] although one study found that there is no relation between the competitive pressure and the adoption of cloud computing technology [19].

H9: competitive pressure has a positive impact on cloud adoption.

\subsubsection{External support}

External support in this research is defined as support from the cloud service provider to influence clients to adopt cloud technology. There is a lack of understanding about cloud services issues such as cloud architecture and pricing models [38]. This could represent one of the barriers for SMEs to adopt cloud computing. CSPs can provide knowledge and expertise to their clients [39].

H10: External support has a positive impact on cloud adoption.

\subsubsection{Government support}

Government support in this context is understood as the regulation, policies and initiatives that support enterprises in the adoption of cloud computing. Government regulation can play an important role in the adoption of technology innovation [9], [26]. Regulation can encourage or discourage cloud computing adoption [9], [22]. One study found that government regulation had more influence on the adoption of E-business in developing countries comparing with developed countries [27]. A study in KSA found that Saudi SMEs seek the support from the government of Saudi Arabia in relation to technology adoption [43].

H11: Government support has a positive impact on cloud adoption.

\subsection{Diffusion of Innovation Category}

\subsubsection{Relative advantage}

Relative advantage is defined as "the degree to which an innovation is perceived as being better than the idea it superseded" [40]. Cloud computing offers technical as well as economic advantages over traditional IT environments.

H12: relative advantage has a positive impact on cloud adoption.

\subsubsection{Compatibility}

Compatibility refers to which extent the new innovation fits with existing organisation's values, culture and practices [9], [40]. From a technical perspective, the extent to which cloud solutions are compatible with existing systems is a key factor when considering moving to a cloud environment. In addition, the extent to which cloud computing services are compatible with statutory regulation is crucial for organisation when considering moving to cloud computing. 
H13: compatibility has a negative impact on cloud adoption.

\subsubsection{Complexity}

Complexity in DOI terms was defined as "the degree to which an innovation is perceived as relatively difficult to understand and use" [44]. Cloud computing comes with some challenges including security and privacy and the use of advanced technology; adopting cloud computing requires new skills and expertise to manage cloud solutions [9]. Thus, these issues will affect cloud adoption.

H14: complexity has a negative impact on cloud adoption.

\section{RESEARCH METHOD}

In order to test the hypothesis in a technically developing country, a web questionnaire was used with computing professionals in KSA. The questionnaire was administered in English and in Arabic. The sample frame refers to the set of people/enterprises from the targeted population that have the change to be selected [41]. In order to select the respondents, two approaches were used to select the sample. Two e-services provider in KSA agreed to distribute questionnaire to their clients. The CSPs used included ELM which is one of the leading CSPs in KSA. To avoid limiting the sample to respondents from CSPs, the questionnaire was also distributed using a professional network. LinkedIn is a professional social network that brings together users with the same interests.

Piloting the questionnaire is a process to test the questionnaire reliability, validity and error testing [42]. To enhance the validity of the questionnaire, a pilot study was conducted in two phases. The first stage of the pilot was conducted to test the understandability of the questionnaire and to ensure the different language versions had the same meaning. In addition, to what extent the questionnaire is readable with different devices (laptop, IPad and Mobile) was examined in this stage. The questionnaire was piloted with eight IS professionals from industry and academia who speak Arabic and English fluently. The second phase of the pilot study was conducted with the CSP. The ELM marketing team who have experience in questionnaire design and analysis evaluated the questionnaire. Some changes were made following the feedback from ELM, including changing some phrasing in Arabic to ensure understandability.

Following the pilot stage, a web based questionnaire platform was used. The questionnaire was built by using Survey Monkey with two version one in Arabic and one in English. The link was circulated by the CSPs to their clients and made available via LinkedIn. At the questionnaire deadline, 103 questionnaires had been completed. Following data cleansing, 81 valid

questionnaires were selected for analysis, representing 81 companies. Reasons for excluding questionnaires from analysis included errors in completion which undermined the validity of the response and too man 'don't know' or blank answers for analysis.

\subsection{Data analysis}

The first set of questions were designed to identify the characteristics of the enterprises which took part in the survey as this study aims to investigate cloud computing adoption from 
organisation level. The graph below shows the number of enterprises that participated in this survey in terms of the industry sectors that belong to it.

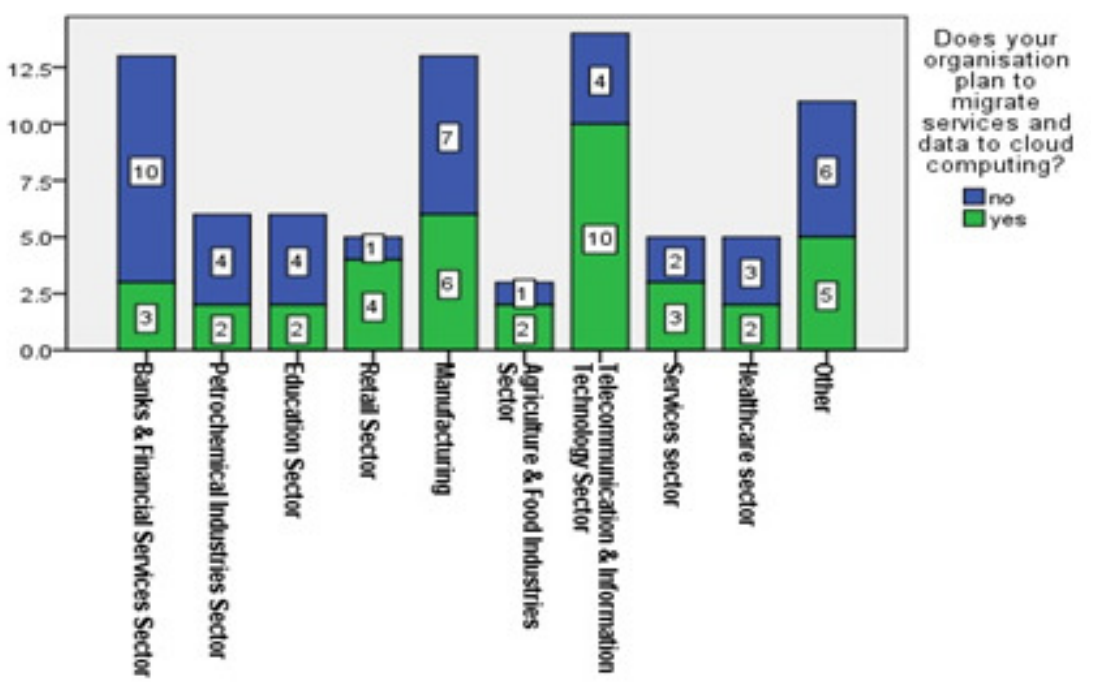

Figure 1: The number of cloud adoption among the industry

In addition, participants were asked to indicate whether their enterprises work on private or government sectors. More than half of respondents belong to the government sectors while about $47 \%$ of participants belong to private sector.

Table 1 illustrates the distribution of enterprises according to its size and status. In addition, the table explains the percentage of enterprise that adopted or plan to adopt cloud computing services.

Table 1. profile of enterprises

\begin{tabular}{|l|l|l|l|l|}
\hline Variable & Group & Frequency & Percentage & Cloud adopter \\
\hline \multirow{5}{*}{ Size } & Small & 18 & $22.2 \%$ & $55.6 \%$ \\
\cline { 2 - 5 } & Medium & 13 & $16 \%$ & $69.2 \%$ \\
\cline { 2 - 5 } & Large & 50 & $61.7 \%$ & $40 \%$ \\
\hline \multirow{3}{*}{ Sector } & Government & 43 & $53.1 \%$ & $48.7 \%$ \\
\cline { 2 - 5 } & Private e & 38 & $46.9 \%$ & $51.3 \%$ \\
\hline \multirow{2}{*}{$\begin{array}{l}\text { Enterprise } \\
\text { status }\end{array}$} & Start-up & 20 & $24.7 \%$ & $85 \%$ \\
\cline { 2 - 5 } & Established & 55 & $67.9 \%$ & $40.1 \%$ \\
\cline { 2 - 5 } & Don't know & 6 & $7.2 \%$ & $0.0 \%$ \\
\hline
\end{tabular}

\subsection{Inferential Analysis}

Logistic regression was used to test the hypothesis described in section. The independent variables are the fourteen hypothesis where is the dependent variable is cloud computing adoption, a binary values were used which is 1 if the firm adopt or plan to adopt cloud computing and 0 otherwise. The independent variables are shown on the table below. 
Table 2. Logistic Regression Results

\begin{tabular}{|c|c|c|c|c|c|c|}
\hline & B & S.E. & Wald & df & Sig. & $\operatorname{Exp}(B)$ \\
\hline Technology readiness & .391 & .780 & .252 & 1 & .616 & 1.479 \\
\hline Security concerns & -1.147 & .435 & 6.951 & 1 & .008 & .318 \\
\hline Technology Barriers & .459 & .397 & 1.336 & 1 & .248 & 1.583 \\
\hline Organisational readiness & 1.989 & .946 & 4.425 & 1 & .035 & 7.312 \\
\hline Firm Size & -.900 & .660 & 1.856 & 1 & .173 & .407 \\
\hline Firm Status & -2.936 & 1.218 & 5.811 & 1 & .016 & .053 \\
\hline Industry Sector & .619 & .888 & .485 & 1 & .486 & 1.856 \\
\hline pp Management Support & 1.768 & .546 & 10.476 & 1 & .001 & 5.858 \\
\hline Competitive pressure & -.176 & .935 & .035 & 1 & .851 & .839 \\
\hline External support & .396 & .920 & .185 & 1 & .667 & 1.486 \\
\hline Government support & -1.774 & .699 & 6.453 & 1 & .011 & .170 \\
\hline Relative advantage & .606 & .900 & .454 & 1 & .501 & 1.833 \\
\hline Compatibility & 1.039 & .507 & 4.202 & 1 & .040 & 2.827 \\
\hline Complexity & -1.080 & .751 & 2.069 & 1 & .150 & .340 \\
\hline Constant & -3.611 & 3.852 & .878 & 1 & .349 & .027 \\
\hline
\end{tabular}

After coding the hypothesis and calculate the mean of the items for each hypothesis, the binary logistic regression run. The independent variable is significant when the $\mathrm{P}$ value is less than 0.05 [43]. Consequently, it can be seen from the data in Table \# that there are six predictors were found statistically significant which are security concerns, organisational readiness, firm status, top management support, government support and compatibility. Three of them belong to the organisational readiness group.

\section{DISCUSSION}

\subsection{Technological context}

Among the three dimensions of technological context, in this research, security was found to have a statistically significant relationship with cloud adoption. Thus, security concerns have a negative impact of adopting cloud computing. This is consistent with the findings in the earlier literature on cloud computing adoption although a 2014 study found that security was not a significant factor in cloud computing adoption; that study suggested that this was due to improvements in security compared to the earlier phase of cloud computing [10]. Unexpectedly, in this study, technology readiness and technology barriers were not found statistically significant. This result are consistent with a 2011 study in a technologically developing country which found that technology readiness were not significant predictor [48] although the 2011 findings were different to those of a study conducted in a technically developed country[10]. One possible explanation for this might be that there is a difference between attitudes of decision makers in technologically developed countries and technologically developing countries. 


\subsection{Organisational context}

Interestingly, in this investigation, three predictors, organisation readiness, top management support and enterprise status, were found to significantly influence the decision about cloud computing adoption. In the literature, only one study was found which identified organisation readiness as a factor that influences cloud computing adoption [20]; that study identified a significant relationship between organisation readiness and cloud adoption which is similar to the findings of this study. In terms of enterprise status, based on the literature review there is no prior study examine firm status empirically in relation to cloud adoption or technology adoption in general. In this study, start-up enterprises were found to be more likely to adopt cloud computing. This may reflect the availability of infrastructure within KSA as established companies will already have developed infrastructure. Top management support was found to be a more significant factor influencing cloud adopting with $\mathrm{P}$ value at 0.001 . The finding on top management support is in line with the findings of previous studies [9], [20], [44]-[46]. Contrary to expectations, this research did not find a significant relationship between the enterprise size and industry sectors. This is in contrast to earlier findings [9], [45], [46] which found a significant relationship between cloud adoption and enterprise size.

\subsection{Environmental context}

The environmental context was examined from three dimensions namely competitive pressure, external support and government support. The only factor found to be statistically significant is the government support. This finding is different from that of [9], [44]. These differences can be explained by the fact that Governments in technological developing counties play a major role in supporting enterprises in adopting new technology in terms of regulation and initiatives [47], [48].

One unanticipated finding was that external support did not significantly affect cloud adoption. This differs from the results of [19]who found computer supplier support has a significant effect on cloud adoption. However, the study conducted by [19], was carried out in a technically developed country. In the research presented here, competitive pressure was not found to a significant factor influencing the adoption of cloud technology. . This result is in agreement with those obtained by [9], [45]. In contrast, [23] and [48] found that the competitive pressure has a significant impact in association with cloud computing. A possible explanation for this is that the studies that found the competitive pressure has a significant factor were limited to one industry sector like [46] or limited to a small number of industry sectors like [20]. Table 3 summarises the findings of this research in respect of the hypothesis developed through the TOE and DOI.

\subsection{Diffusion of Innovation Category}

Unexpectedly, relative advantage was not found to be a significant factor in this study. This contrasts with findings from previous studies [9], [20], [44]-[46] which identified a significant relationship between relative advantage and cloud computing. An important finding in this research was that compatibility has a significant impact on cloud computing adoption. This finding is in accord with recent studies which indicated that the compatibility has a positive impact on cloud adoption [20], [45] although some studies report different findings [9], [44], [46]. Finally, it is somewhat surprising that in this study complexity was not found to significantly affect cloud adoption. This result is supported by other studies [44], [46] but differs from that of recent studies that found complexity negatively influences cloud computing adoption [9], [20], [45]. It should be noted that the respondents in this study had technical backgrounds. 
Table 3. Summary of results

\begin{tabular}{|l|l|l|}
\hline \multicolumn{2}{|l|}{ Technological context } & Rejected \\
\hline H1 & Technology readiness & Accepted \\
\hline H2 & Security concerns & Rejected \\
\hline H3 & Technology Barriers & \multicolumn{2}{|l|}{} \\
\hline Organisational context & Accepted \\
\hline H4 & Organisational readiness & Rejected \\
\hline H5 & Firm Size & Accepted \\
\hline H6 & Firm Status & Rejected \\
\hline H7 & Industry Sector & Accepted \\
\hline H8 & Top Management Support & \\
\hline Environmental context & Rejected \\
\hline H9 & Competitive pressure & Rejected \\
\hline H10 & External support & Accepted \\
\hline H11 & Government support & \\
\hline Diffusion of innovation & Rejected \\
\hline H12 & Relative advantage & Accepted \\
\hline H13 & Compatibility & Rejected \\
\hline H14 & Complexity & \\
\hline
\end{tabular}

\section{CONCLUSION}

This study integrates the DOI and TOE framework to investigate the factors that affect cloud computing adoption decision in Saudi Arabia. From the fourteen hypothesis were developed, there are only six hypothesis were found statistically significant. These factors are security concerns, organisation readiness, top management support, firm status, government support and compatibility. When evaluated against the literature on cloud computing adoption, it appears that security concerns and government support are more influential factors in technologically developing countries than in technologically developed countries. We found that start up companies were more likely to adopt cloud computing solutions. This may reflect the pricing model of cloud computing but may also reflect infrastructure differences between technically developed and technically developing countries. In addition,

The findings of this study contributes to both academia and industry. Cloud service providers can use the findings of this study to support marketing and decision making. The investigation shows that government has a major role to play in encouraging enterprises to adopt technology in general and specifically cloud computing in technologically developing countries. This study adds the body of knowledge about cloud computing adoption specifically in technologically developing countries.

\section{ACKNOWLEDGEMENT}

The author would like to thank ELM for distributing the questionnaire and piloting the questionnaire. 


\section{REFERENCES}

[1] S. Rajan and A. Jairath, "Cloud Computing: The Fifth Generation of Computing," in 2011 International Conference on Communication Systems and Network Technologies, 2011, pp. 665-667.

[2] K. Ruan, J. Carthy, and T. Kechadi, "Cloud forensics: An overview," ... Digit. forensics VII, vol. 0, 2011.

[3] E. O. Yeboah-Boateng and K. A. Essandoh, "Factors Influencing the Adoption of Cloud Computing by Small and Medium Enterprises in Developing Economies," Int. J. Emerg. Sci. Eng., no. 4, pp. 1320, 2014.

[4] S. T. Alharbi, "Users' Acceptance of Cloud Computing in Saudi Arabia," Int. J. Cloud Appl. Comput., vol. 2, no. 2, pp. 1-11, 2012.

[5] F. C. Cua, "Information Systems Theory," in Information Systems Theory: Explaining and Predicting Our Digital Society, vol. 28, Y. K. Dwivedi, M. R. Wade, and S. L. Schneberger, Eds. New York, NY: Springer New York, 2012, pp. 303-333.

[6] B. Ramdani, D. Chevers, and D. a. Williams, "SMEs\&apos; adoption of enterprise applications: A technology-organisation-environment model,” J. Small Bus. Enterp. Dev., vol. 20, no. 4, pp. 735753, 2013.

[7] H. Gangwar, H. Date, and A. D. Raoot, "Review on IT adoption: insights from recent technologies," J. Enterp. Inf. Manag., vol. 27, no. 4, pp. 488-502, Jul. 2014.

[8] M. Nkhoma and D. Dang, "Contributing factors of cloud computing adoption: a technologyorganisation-environment framework approach,” Int. J. Inf. ..., vol. 1, no. 1, pp. 38-49, 2013.

[9] T. Oliveira, M. Thomas, and M. Espadanal, "Assessing the determinants of cloud computing adoption: An analysis of the manufacturing and services sectors," Inf. Manag., vol. 51, no. 5, pp. 497-510, Jul. 2014.

[10] A. Rahimli, "Factors influencing organization adoption decision on cloud computing," Int. J. Cloud Comput. Serv. Sci., vol. 2, no. 2, pp. 140-146, 2013.

[11] S. S. Bharadwaj and P. Lal, "Exploring the impact of Cloud Computing adoption on organizational flexibility: A client perspective," in 2012 International Conference on Cloud Computing Technologies, Applications and Management (ICCCTAM), 2012, pp. 121-131.

[12] R. Ramanathan, U. Ramanathan, and L. W. L. Ko, “Adoption of RFID technologies in UK logistics: Moderating roles of size, barcode experience and government support," Expert Syst. Appl., vol. 41, no. 1, pp. 230-236, Jan. 2014.

[13] H.-F. Lin and S.-M. Lin, "Determinants of e-business diffusion: A test of the technology diffusion perspective," Technovation, vol. 28, no. 3, pp. 135-145, Mar. 2008.

[14] J. Tan, K. Tyler, and A. Manica, "Business-to-business adoption of eCommerce in China," Inf. Manag., vol. 44, no. 3, pp. 332-351, Apr. 2007.

[15] G. Feuerlicht and N. Margaris, "Cloud Computing Adoption : A comparative study," pp. 441-448, 2007.

[16] P. Gottschalk and H. Solli-Sæther, "Critical success factors from IT outsourcing theories: an empirical study,” Ind. Manag. Data Syst., vol. 105, no. 6, pp. 685-702, 2005.

[17] I. Gonzenbach, C. Russ, and J. vom Brocke, "Make or Buy? Factors that Impact the Adoption of Cloud Computing on the Content Level," in Enterprise Content Management in Information Systems Research, J. vom Brocke and A. Simons, Eds. Berlin, Heidelberg: Springer Berlin Heidelberg, 2014, pp. $145-161$.

[18] M. Carroll, A. van der Merwe, and P. Kotze, "Secure cloud computing: Benefits, risks and controls," in 2011 Information Security for South Africa, 2011, pp. 1-9.

[19] G. Chao, A. Peng, A. Dutta, and A. Choudhary, "Exploring Critical Risks Associated with Enterprise Cloud Computing," in CloudComp, vol. 133, V. C. M. Leung and M. Chen, Eds. Cham: Springer International Publishing, 2014, pp. 132-141.

[20] H. Gangwar, H. Date, and R. Ramaswamy, "Understanding determinants of cloud computing adoption using an integrated TAM-TOE model,” J. Enterp. Inf. Manag., vol. 28, no. 1, pp. 107-130, Feb. 2015. 
[21] N. Phaphoom, X. Wang, S. Samuel, S. Helmer, and P. Abrahamsson, "A Survey Study on Major Technical Barriers Affecting the Decision to Adopt Cloud Services," J. Syst. Softw., vol. 103, pp. 167-181, Feb. 2015.

[22] J. Baker, "The Technology-Organization-Environment Framework," in Information Systems Theory: Explaining and Predicting Our Digital Society, vol. 28, Y. K. Dwivedi, M. R. Wade, and S. L. Schneberger, Eds. New York, NY: Springer New York, 2012, pp. 231-245.

[23] M. G. Aboelmaged, "Predicting e-readiness at firm-level: An analysis of technological, organizational and environmental (TOE) effects on e-maintenance readiness in manufacturing firms," Int. J. Inf. Manage., vol. 34, no. 5, pp. 639-651, Oct. 2014.

[24] W.-Y. Pan, Ming-Ju and Jang, "DETERMINANTS OF THE ADOPTION OF ENTERPRISE RESOURCE PLANNING WITHIN THE TECHNOLOGY ORGANIZATION ENVIRONMENT FRAMEWORK : TAIWAN' S COMMUNICATIONS INDUSTRY,” J. Comput. Inf. Syst., vol. 48, no. 3, 2008.

[25] K. Zhu, K. Kraemer, and J. Dedrick, "Information technology payoff in e-business environments: an international perspective on value creation of e-business in the financial services industry," J. Manag. Inf. Syst., vol. 21, no. 1, pp. 17-54, 2004.

[26] K. Zhu, K. L. Kraemer, and S. Xu, "The Process of Innovation Assimilation by Firms in Different Countries: A Technology Diffusion Perspective on E-Business,” Manage. Sci., vol. 52, no. 10, pp. 1557-1576, Oct. 2006.

[27] H.-M. Lai, I.-C. Lin, and L.-T. Tseng, "High-level managers' considerations for RFID adoption in hospitals: an empirical study in Taiwan.," J. Med. Syst., vol. 38, no. 2, p. 3, Feb. 2014.

[28] M. Swink, "Technological Innovativeness as a Moderator of New Product Design Integration and Top Management Support,” J. Prod. Innov. Manag., vol. 17, no. 3, pp. 208-220, May 2000.

[29] C.-H. Yeh, G.-G. Lee, and J.-C. Pai, "Using a technology-organization-environment framework to investigate the factors influencing e-business information technology capabilities," Inf. Dev., Feb. 2014.

[30] A. Jeyaraj, J. W. Rottman, and M. C. Lacity, "A review of the predictors, linkages, and biases in IT innovation adoption research,” J. Inf. Technol., vol. 21, no. 1, pp. 1-23, Jan. 2006.

[31] B. Ramdani, D. Chevers, and D. a. Williams, "SMEs' adoption of enterprise applications: A technology-organisation-environment model," J. Small Bus. Enterp. Dev., vol. 20, no. 4, pp. 735753, 2013.

[32] A. N. Riyadh, S. Akter, and N. Islam, "The Adoption of E-banking in Developing Countries: A Theoretical Model for SMEs,” Int. Rev. Bus. Res. Pap., vol. 5, no. 6, pp. 212-230, 2009.

[33] M. N. O. Sadiku, S. M. Musa, and O. D. Momoh, "Cloud Computing: Opportunities and Challenges," IEEE Potentials, vol. 33, no. 1, pp. 34-36, Feb. 2014.

[34] P. Gupta, a. Seetharaman, and J. R. Raj, "The usage and adoption of cloud computing by small and medium businesses,” Int. J. Inf. Manage., vol. 33, no. 5, pp. 861-874, Oct. 2013.

[35] I. Son, D. Lee, J. Lee, and Y. Chang, "Understanding the impact of it service innovation on firm performance: The case of Cloud Computing," in PACIS 2011 Proceedings, 2011.

[36] S. Srinivasan, "Basic Cloud Computing Types," in Cloud Computing Basics, New York, NY: Springer New York, 2014, pp. 17-41.

[37] A. Khajeh-Hosseini, I. Sommerville, and I. Sriram, "Research challenges for enterprise cloud computing," arXiv Prepr. arXiv1001.3257, 2010.

[38] S. C. Misra and A. Mondal, "Identification of a company's suitability for the adoption of cloud computing and modelling its corresponding Return on Investment," Math. Comput. Model., vol. 53, no. 3-4, pp. 504-521, Feb. 2011.

[39] P. Ifinedo, "Internet/e-business technologies acceptance in Canada's SMEs: an exploratory investigation," Internet Res., vol. 21, no. 3, pp. 255-281, Jan. 2011.

[40] E. M. Rogers, Diffusion of innovations, 5th ed. New York, 2003.

[41] J. Fowler and J. Floyd, Survey research methods. Sage publications, 2008.

[42] I. Brace, Questionnaire design : how to plan, structure and write survey material for effective market research, 3rd ed. London N1 9JN: Kogan Page Limited, 2013.

[43] J. H. McDonald, HANDBOOK OF BIOLOGICAL STATISTICS. Baltimore: Sparky House, 2009. 
[44] H. P. Borgman, B. Bahli, H. Heier, and F. Schewski, "Cloudrise: Exploring Cloud Computing Adoption and Governance with the TOE Framework," in 2013 46th Hawaii International Conference on System Sciences, 2013, pp. 4425-4435.

[45] Y. Alshamaila, S. Papagiannidis, and F. Li, "Cloud computing adoption by SMEs in the north east of England: A multi-perspective framework," J. Enterp. Inf. Manag., vol. 26, no. 3, pp. 250-275, 2013.

[46] C. Low, Y. Chen, and M. Wu, "Understanding the determinants of cloud computing adoption," Ind. Manag. Data Syst., vol. 111, no. 7, pp. 1006-1023, 2011.

[47] R. Alghamdi, S. Drew, and S. Alkhalaf, "Government Initiatives : The Missing Key for E-commerce Growth in KSA," World Acad. Sci. Eng. Technol., vol. 77, pp. 772-775, 2011.

[48] R. AlGhamdi, A. Nguyen, J. Nguyen, and S. Drew, "Factors influencing E-commerce Adoption by Retailers in Saudi Arabia. arXiv preprint," Int. J. Electron. Commer. Stud., vol. 3, no. 1, pp. 83-100, 2012. 